\title{
Using bugs as drugs: Microbial ecosystem therapeutics
}

\author{
Allen-Vercoe $E^{1 *}$, Petrof $E^{2}$ \\ ${ }^{1}$ Department of Molecular and Cellular Biology, University of Guelph, Guelph, ON \\ ${ }^{2}$ Department of Medicine, Queen's University, Kingston, ON \\ *Correspondence: eav@uoguelph.ca
}

\begin{abstract}
The human gut harbours a dense and highly diverse microbial ecosystem-the microbiota-that plays an important role in the maintenance of health. Modern lifestyle practices, including widespread antibiotic use, have degraded microbiota diversity, compromising the integrity of this vital ecosystem and creating susceptibility to diseases such as Clostridium difficile infection. Treatment of patients to restore the diversity of the gut microbiota offers a logical solution to disease. Although fecal microbial therapy (FMT) has started to gain traction as an effective method to effect this restoration, it is not without risks and there are significant barriers to its implementation in the clinic. Some of the risks and challenges with FMT are addressed by microbial ecosystem therapeutics (MET), an alternative approach to FMT that uses selected, defined microbial ecosystems to redress microbiota balance and functionality. The time has come for the use of bugs as drugs.
\end{abstract}

\section{Introduction}

Human beings are colonized by trillions of microbes; every skin and mucosal surface houses a diverse community of microbial cells (1). The gut contains by far the greatest density and diversity of microbes in the human body: the colon contains up to 1,012 microbial cells per gram (2), a microbiota made up largely of bacteria, but also including smaller numbers of archaea (prokaryotes distinct from bacteria), yeasts and protists (eukaryotic microbes) (3). Although some opportunistic pathogens reside among this microbiota, the vast majority of species within the ecosystem are either benign, or more commonly are beneficial, carrying out a great many functions for us that aid in the maintenance of health. Such functions include modulation of the immune system, production of beneficial substrates such as butyrate (which acts as a food source for colonocytes, among other health-promoting attributes) and vitamins, and production of certain chemical signaling molecules that may play important roles in the control of pathogenic exposure to the host $(4,5)$.

Despite this plethora of functions, modern medicine has, until fairly recently, ignored the importance of the gut microbiota, and in fact has fostered the use of antibiotics for treatment of infections. As well as encouraging antimicrobial resistance, widespread antibiotic use has inadvertently impaired the integrity of the gut microbiota through collateral damage (6). In fact, it has been theorized that such ecosystem damage and subsequent loss of microbial species diversity may be a major contributing factor towards the increase of several chronic diseases that currently plague modern society (7). Certainly this theory is supported by a growing weight of evidence demonstrating reduction of the diversity of the gut microbiota associated with many diseases from inflammatory bowel disease to psoriatic arthritis $(8,9)$. However, because the average gut microbiota contains a large number of microbial species, and because the species profile of a given individual is distinct and unique (10), trying to define the underlying mechanisms of these diseases as they relate to the microbiota is challenging. 
In this overview we describe how depletion of ecosystem diversity may lead to disease, using the example of Clostridium difficile infection (CDI). We also discuss how strategies to replace lost ecosystem diversity using fecal microbes can be useful in the treatment of $C D I$, and how the risks and challenges of this approach can be mitigated by the use of defined microbial ecosystems.

\section{Treating recurrent Clostridium difficile infection}

There is one condition that is clearly understood to be a result of gut ecosystem disturbance and lack of microbial diversity - this is CDI. A persistent problem in the hospital environment, CDI is usually a complication of broad spectrum antibiotic use, where these antibiotics reduce the diversity of the gut microbiota of the patient; with this protective diversity gone, $C$. difficile can grow unchecked, to high numbers (11). As well as antibiotic use, other interventions known to decrease gut microbial diversity, such as the use of proton pump inhibitors (PPIs), or chemotherapy, may also contribute to the risk of developing CDI (12-14). Once a particular growth threshold has been exceeded, $C$. difficile produces a range of exotoxins with damaging effects on human colonocytes, leading to profuse diarrhea that can progress to pseudomembranous colitis, toxic megacolon and, in severe cases, death (11).

The current standard therapeutic solution to this problem is to apply more antibiotics. Drugs such as metronidazole or oral vancomycin are used, which have activity against $C$. difficile, to reduce the numbers of the pathogen and hence the toxigenic load (15). However, there is some backwards logic here; the CDI patient suffers from a lack of gut microbial diversity, usually as a result of antibiotic use. Further antibiotic use may have a short-term benefit as the numbers of the pathogen may be temporarily reduced, but C. difficile is an endospore-forming bacterium that can sporulate when faced with an inhospitable environment (such as the presence of antibiotics). The spores are resistant to metronidazole and oral vancomycin, allowing $C$. difficile to germinate and cause problems once more when the antibiotic is removed (16). Meanwhile, antibiotic exposure removes the natural resistance to $C$. difficile colonization-the gut microbiota. CDI patients increasingly get caught up in a cycle of $C$. difficile-suppressive antibiotic use, which has negative effects on the protective gut microbiota, preventing recovery from infection and leading to recurrent CDI. This problem is a big one-a recent survey across the United States carried out in 2011 estimated half a million cases of CDI for that year (17). Recurrent CDI accounts for $10 \%$ to $20 \%$ of cases (18).

An alternative to antibiotic treatment for recurrent CDI lies in restitution of the diversity of the gut microbiota. Re-establishing this protective diversity displaces $C$. difficile, rather like how re-seeding a damaged lawn with healthy new turf can displace the weeds. At its most primitive, this is achieved through use of "fecal microbial therapy" (FMT), literally a transfer of stool from a healthy donor into a patient by way of rectal enema, colonoscopy, or even nasoduodenal tube (19). The approach is not new; practitioners of Chinese medicine used preparations of fecal microbes to treat diseases such as dysentery as early as the $4^{\text {th }}$ century BC, and veterinarians have used the principle of fecal transplant to treat animals with gastrointestinal problems for decades. Whilst the practice is unpleasant, it is clearly effective; $81 \%$ of patients provided FMT for recurrent CDI were rapidly cured of their infections with one dose of FMT, compared to $31 \%$ given vancomycin in a recent clinical trial (20). However, FMT is not without risk; although when done under medical supervision donors are rigorously screened for known pathogens potentially passed on through stool, there is so much diversity within the human gut microbiota, with many as yet uncharacterized microbial species, there is no way to currently know whether unknown pathogens are being transferred by the practice.

\section{Challenges with FMT}

Although FMT is a promising alternative to antibiotic use for treatment of recurrent CDI, it is difficult to regulate. Regulation is problematic because each donor's stool can be considered as a different, complex therapeutic (21). As yet there is no current consensus on how to define and standardize the treatment, although the use of frozen stool products and "super donors" goes some way to address the issue (19). A further complication is that government-sanctioned administrative oversight of the procedure may limit its availability and therefore has the potential to drive the practice underground; FMT can be performed at home 
with no medical supervision, using materials that can be easily procured from a drug store. Recently, Health Canada produced a guidance document Regulation of Fecal Microbiota Therapy for the Treatment of C. difficile Infections to inform FMT practitioners of the government stance on the practice of FMT (22); Health Canada views stool used therapeutically as a biologic drug, because it has been derived from a human source (21).

There are also concerns about the long-term safety of FMT. While for recurrent CDI the short-term benefits are clear, so far there has been a dearth of studies of the long-term effects of the treatment. And while such negative effects may be negligible for elderly patients, CDI is becoming increasingly common in younger, otherwise healthy people (23). So, how should we proceed with this promising therapy, while also ensuring safety?

\section{Microbial ecosystem therapeutics}

The challenges of FMT can be addressed by the development of standardized "microbial ecosystem therapeutics" (MET). MET can be thought of as a new type of "probiotic," one in which beneficial microbes are carefully selected from a single healthy donor, thoroughly screened for any potential detrimental attributes (e.g., antibiotic resistance) and then recombined into an ecosystem. Microbes within ecosystems tend to work together synergistically, and we hypothesize that our approach capitalizes on this microbial synergism, enhancing any beneficial effects. Our prototype product, MET-1 (RePOOPulate), which was shown to be effective as treatment for recurrent CDI in a small proof-of-principle trial, was created through careful culture of the diversity from the stool of a healthy woman (24). From this pool of isolates, a subset of 33 strains was selected, each with minimal antibiotic resistance and lacking any known virulence determinants; these were formulated into an ecosystem and tested for functional integrity in vitro using chemostat technology.

The resulting, defined microbial ecosystem has many advantages over FMT as a therapeutic, including the ability to standardize the manufacturing process and to create a quality controlled product, one of the key guidelines for regulation of a biologic drug. This product can potentially be delivered orally as a live, freeze-dried preparation, simplifying treatment. Perhaps the greatest advantage of this approach over the use of stool is the fact that the long-term effects of the treatment may be more easily studied such that, as with any biologic drug, safety of the product can be increasingly ensured over time. MET-1 is the first in a series of MET products currently being created to provide treatment options tailored to patient lifestyle-for example, diet, known to be a key driver of ecosystem diversity (25). While the regulatory hurdles to bring these products to market are high, the expected benefits are higher, and the ability to track introduced microbes in a patient may also help to answer key ecological questions about, for example, the influence of the host on ecosystem function and stability. However, it is important to understand that development of the MET principle is still in its infancy and there are many questions that still need to be answered-for example, what are the essential components of MET ecosystems that allow for their therapeutic benefit? How important is functional redundancy of a MET ecosystem to its efficacy in the treatment of disease? Do introduced MET microbes colonize the host indefinitely, or do they simply act as a "band aid" to allow recovery of the recipient's original ecosystem? Are there any detrimental effects of MET in comparison to FMT?

\section{Conclusion}

We now have the scientific basis to use bugs as drugs. FMT has documented benefit, but it also has its challenges. We believe that MET represents a new chapter in medicine. In this new era, microbes will finally be considered as our allies, and their properties, such as their antivirulence determinants, may be leveraged for the preservation of health.

In the future we should consider the use of MET in other diseases where adverse compositional changes in the gut microbiota may be a key factor in disease. There are indications in the literature, for example, that FMT-based approaches may have an impact in such diseases as ulcerative colitis, obesity and 
metabolic syndrome $(8,26-29)$. What is needed is the introduction of tailored METs developed to address the underlying gut microbial dysbiosis in these diseases, and to monitor the outcomes of MET-based intervention in well-designed clinical trials.

\section{Conflict of interest}

The authors are co-founders of Nubyota LLC, a company created to commercialize the application of microbial ecosystem therapeutics in medicine.

\section{References}

(1) Human Microbiome Project C. Structure, function and diversity of the healthy human microbiome. Nature. 2012;486(7402):207-14.

(2) O'Hara AM, Shanahan F. The gut flora as a forgotten organ. EMBO Reports. 2006;7(7):688-93.

(3) Rajilic-Stojanovic M, de Vos WM. The first 1000 cultured species of the human gastrointestinal microbiota. FEMS Microbiology Reviews. 2014;38(5):996-1047.

(4) Frick JS, Autenrieth IB. The gut flora and its variety of roles in health and disease. Current Topics in Microbiology and Immunology. 2013;358:273-89.

(5) Antunes LC, McDonald JA, Schroeter K, et al. Antivirulence activity of the human gut metabolome. mBio. 2014; $\mathrm{e} 01183-01114$.

(6) Modi SR, Collins JJ, Relman DA. Antibiotics and the gut microbiota. The Journal of Clinical Investigation. 2014;124(10):4212-8.

(7) Blaser MJ, Falkow S. What are the consequences of the disappearing human microbiota? Nature Reviews Microbiology. 2009 Dec;7(12):887-94

(8) Matsuoka K, Kanai T. The gut microbiota and inflammatory bowel disease. Seminars in Immunopathology. 2015;37(1):47-55.

(9) Scher JU, Ubeda C, Artacho A, et al. Decreased bacterial diversity characterizes the altered gut microbiota in patients with psoriatic arthritis, resembling dysbiosis in inflammatory bowel disease. Arthritis \& Rheumatology. 2015;67(1):128-39.

(10) Sekirov I, Russell SL, Antunes LC, Finlay BB. Gut microbiota in health and disease. Physiological Reviews. 2010;90(3):859-904.

(11) Awad MM, Johanesen PA, Carter GP, Rose E, Lyras D. Clostridium difficile virulence factors: Insights into an anaerobic spore-forming pathogen. Gut Microbes. 2014;5(5):579-93.

(12) Freedberg DE, Lebwohl B, Abrams JA. The impact of proton pump inhibitors on the human gastrointestinal microbiome. Clinics in Laboratory Medicine. 2014;34(4):771-85.

(13) McDonald EG, Milligan J, Frenette C, Lee TC. Continuous proton pump inhibitor therapy and the associated risk of recurrent Clostridium difficile infection. 2015; JAMA Internal Medicine. 2015 May 1;175(5):784-91.

(14) Zwielehner J, Lassl C, Hippe B, et al. Changes in human fecal microbiota due to chemotherapy analyzed by TaqMan PCR, 454 sequencing and PCR-DGGE fingerprinting. PloS One. 2011;6(12):e28654.

(15) Soriano MM, Johnson S. Treatment of Clostridium difficile infections. Infectious Disease Clinics of North America. 2015;29(1):93-108.

(16) Barra-Carrasco J, Paredes-Sabja D. Clostridium difficile spores: A major threat to the hospital environment. Future Microbiology. 2014;9(4):475-86.

(17) Lessa FC, Mu Y, Bamberg WM, et al. Burden of Clostridium difficile infection in the United States. New Engl J Med. 2015;372(9):825-34.

(18) Surawicz CM. Clostridium difficile infection: Risk factors, diagnosis and management. Current Treatment Options in Gastroenterology. 2015;13(1):121-9.

(19) Merenstein D, El-Nachef N, Lynch SV. Fecal microbiology therapy: Promises and pitfalls. Journal of Pediatric Gastroenterology and Nutrition. 2014;59(2):157-61.

(20) van Nood E, Vrieze A, Nieuwdorp M, et al. Duodenal infusion of donor feces for recurrent Clostridium difficile. New Engl J Med. 2013;368(5):407-15.

(21) Allen-Vercoe E, Reid G, Viner N, et al. A Canadian Working Group report on fecal microbial therapy: Microbial ecosystems therapeutics. Canadian Journal of Gastoenterology. 2012;26(7):457-62.

(22) Health Canada. Guidance Document: Regulation of Fecal Microbiota Therapy for the Treatment of $C$. difficile Infections. Ottawa: Health Canada; 2015. http://www.hc-sc.gc.ca/dhp-mps/consultation/biolog/fecal_microbiota-bacterio_fecale-eng.php

(23) Gupta A, Khanna S. Community-acquired Clostridium difficile infection: An increasing public health threat. Infection and Drug Resistance. 2014;7:63-72.

(24) Petrof EO, Gloor GB, Vanner SJ, et al. Stool substitute transplant therapy for the eradication of Clostridium difficile infection: 'RePOOPulating' the gut. Microbiome. 2013;1:3.

(25) Xu Z, Knight R. Dietary effects on human gut microbiome diversity. British Journal of Nutrition. 2015;113 Suppl:S1-5.

(26) Mondot S, de Wouters T, Doré J, Lepage P. The human gut microbiome and its dysfunctions. Digestive Diseases. 2013;31(3-4):278-85.

(27) Tilg H, Moschen AR. Microbiota and diabetes: An evolving relationship. Gut. 2014;63(9):1513-21.

(28) Alang N, Kelly C. Weight gain after fecal microbiota transplantation. Open Forum Infectious Diseases. 2015;2(1):1-2.

(29) Vrieze A, van Nood E, Hollerman F, et al. Transfer of intestinal microbiota from lean donors increases insulin sensitivity in individuals with metabolic syndrome. Gastroenterology. 2012 Oct;143(4):913-6.e917. 